\title{
GROTHENDIECK GROUPS OF ORDERS IN SEMISIMPLE ALGEBRAS
}

\author{
BY \\ A. HELLER AND I. REINER(1)
}

Introduction. Let $R$ be a noetherian domain with quotient field $F$, and let $A$ be an $R$-algebra which is finitely generated and torsion free as $R$-module. Define the $F$-algebra $A^{*}$ to be $F \otimes_{R} A$. We may form the Grothendieck groups $K^{0}(A)$, $K^{0}\left(A^{*}\right), K_{t}^{0}(A)$, the last of which is obtained from the category of $R$-torsion $A$-modules (see $\$ 1$ for the definitions of these groups).

On the other hand, we may define a Whitehead group $K^{1}\left(A^{*}\right)$. We shall set up a homomorphism $\Delta: K^{1}\left(A^{*}\right) \rightarrow K_{t}^{0}(A)$. If $A^{*}$ is semisimple, we obtain an exact sequence

$$
K^{1}\left(A^{*}\right) \stackrel{\Delta}{\rightarrow} K_{t}^{0}(A) \rightarrow K^{0}(A) \rightarrow K^{0}\left(A^{*}\right) \rightarrow 0 .
$$

This result is applied to the case where $A=R G$, the group r $: \mathrm{g}$ of a finite group $G$ over a Dedekind ring $R$ of characteristic 0 . If $F$ is a splitting field for $G$, we are able to compute $K^{0}(A)$ explicitly in terms of the arithmetic of $R$ and the decomposition matrices of $G$.

In a recent paper [5], Swan (using different methods) has independently obtained a number of striking results on the structure of $K^{0}(A)$.

Throughout this paper, all rings are left noetherian and have unity elements. All modules are left, finitely generated modules. The ring of rational integers is denoted by $Z$.

1. Grothendieck groups. 1. Let $A$ be a ring, and let $\mathscr{A}$ be the free abelian group generated by the symbols $(M)$, where $M$ ranges over all $A$-modules. Define $\mathscr{A}_{0}$ as the subgroup of $\mathscr{A}$ generated by elements of the form

$$
(M)-\left(M^{\prime}\right)-\left(M^{\prime \prime}\right) \text {, }
$$

where $0 \rightarrow M^{\prime} \rightarrow M \rightarrow M^{\prime \prime} \rightarrow 0$ ranges over all short exact sequences of $A$-modules. Then set $K^{0}(A)=\mathscr{A} / \mathscr{A}_{0}$, the Grothendieck group of $A$. We use [M] to denote the image of $M$ in $K^{0}(A)$.

2. If $A$ is a ring with minimum condition, then the Jordan-Hölder theorem is valid for $A$-modules. Consequently, if $\left\{M_{1}, \cdots, M_{n}\right\}$ is a full set of irreducible $A$-modules, then $K^{0}(A)$ is the free $Z$-module with free $Z$-basis $\left[M_{1}\right], \cdots,\left[M_{n}\right]$.

Presented to the Society, March 25, 1963 under the title On Grothendieck groups of orders; received by the editors April 5, 1963.

(1) The research of the first author was supported in part by the National Science Foundation that of the second author by the Guggenheim Foundation and the Office of Naval Research. 
3. Returning to the general case, we wish to show that if $M$ and $N$ are $A$-modules, then $[M]=[N]$ in $K^{0}(A)$ if and only if $M$ and $N$ have the same composition factors, in some sense. More precisely, we prove

Lemma 1. Let $M$ and $N$ be A-modules. Then $[M]=[N]$ in $K^{0}(A)$ if and only if there exist two exact sequences

$$
0 \rightarrow U \rightarrow M \oplus W \rightarrow V \rightarrow 0,0 \rightarrow U \rightarrow N \oplus W \rightarrow V \rightarrow 0,
$$

for some choice of A-modules $U, V$ and $W$.

Proof. If there exist modules $U, V, W$ for which the sequences in (1) are exact then clearly $[M]=[N]$ in $K^{0}(A)$.

Conversely, suppose that: $[M]=[N]$ in $K^{0}(A)$, and write $K^{0}(A)=\mathscr{A} / \mathscr{A}_{0}$, using the notation of $\S 1.1$. Then

$$
(M)-(N)=\sum_{X} \pm\left\{(X)-\left(X^{\prime}\right)-\left(X^{\prime \prime}\right)\right\},
$$

where $0 \rightarrow X^{\prime} \rightarrow X \rightarrow X^{\prime \prime} \rightarrow 0$ is exact. Therefore

$$
(M)+\sum_{i}\left\{\left(X_{i}^{\prime}\right)+\left(X_{i}^{\prime \prime}\right)\right\}+\sum_{j}\left(Y_{j}\right)=(N)+\sum_{i}\left(X_{i}\right)+\sum_{j}\left\{\left(Y_{j}^{\prime}\right)+\left(Y_{j}^{\prime \prime}\right)\right\}
$$

holds true in $\mathscr{A}$, with $0 \rightarrow X_{i}^{\prime} \rightarrow X_{i} \rightarrow X_{i}^{\prime \prime} \rightarrow 0$ exact for each $i$, and $0 \rightarrow Y_{j}^{\prime} \rightarrow Y_{j} \rightarrow Y_{j}^{\prime \prime} \rightarrow 0$ exact for each $j$. It follows from the definition of $\mathscr{A}$ that any term $(T)$ which occurs on the left-hand side of equation (2) with some multiplicity $t$, say, must also occur on the right-hand side with multiplicity $t$. Set $X=\Sigma^{\oplus} X_{i}, X^{\prime}=\Sigma^{\oplus} X_{i r}$, and so on. The preceding shows that

$$
M \oplus X^{\prime} \oplus X^{\prime \prime} \oplus Y \cong N \oplus X \oplus Y^{\prime} \oplus Y^{\prime \prime} .
$$

Let $W$ be a module isomorphic to both of the above.

Since $W \cong N \oplus X \oplus Y^{\prime} \oplus Y^{\prime \prime}$, there is an embedding of $X^{\prime} \oplus Y^{\prime}$ in $W$ with quotient module $N \oplus X^{\prime \prime} \oplus Y^{\prime \prime}$. Thus there exists an exact sequence

$$
0 \rightarrow X^{\prime} \oplus Y^{\prime} \rightarrow M \oplus W \rightarrow M \oplus N \oplus X^{\prime \prime} \oplus Y^{\prime \prime} \rightarrow 0 .
$$

Analogously, there exists another such exact sequence with $M$ and $N$ interchanged. This completes the proof of the lemma.

4. We next introduce Bass' version of the Whitehead group $K^{1}(A)$ (see [1]). Let $A$ be a ring, and consider the category whose objects are pairs $(M, \mu)$ consisting of an $A$-module $M$ and an automorphism $\mu$ of $M$. By a map $\phi:(M, \mu) \rightarrow(N, v)$ of one such object into another, we mean an element $\phi \in \operatorname{Hom}_{A}(M, N)$ such that $\phi \mu=v \phi$. Consider a sequence

$$
0 \longrightarrow(L, \lambda) \stackrel{\phi}{\longrightarrow}(M, \mu) \stackrel{\psi}{\longrightarrow}(N, v) \longrightarrow 0
$$

of objects and maps in this category. Then the sequence is exact in this category if and only if $0 \rightarrow L \stackrel{\Phi}{\rightarrow} M \stackrel{\leftrightarrow}{\rightarrow} N \rightarrow 0$ is exact in the usual sense. 
(For the orientation of the reader, we remark that if one regards $\phi$ as an embedding of $L$ in $M$, and $\psi$ as the canonical projection of $M$ onto $M / L$, then the exactness of (3) simply means that $\mu$ is an automorphism of $M$ which maps $L$ onto itself, thereby inducing an automorphism $\lambda$ of $L$ and an automorphism $v$ of the factor module $M / L$.)

Let $\mathscr{B}$ be the free abelian group with generators $(M, \mu)$, where $M$ ranges over all $A$-modules, and $\mu$ ranges over all automorphisms of $M$. Define $\mathscr{B}_{0}$ as the subgroup of $\mathscr{B}$ generated by the elements

$$
(M, \mu)-(L, \lambda)-(N, v)
$$

gotten from all exact sequences given by (3), together with all elements of the form

$$
\left(M, \mu \mu^{\prime}\right)-(M, \mu)-\left(M, \mu^{\prime}\right) .
$$

Now let $K^{1}(A)=\mathscr{B} / \mathscr{B}_{0}$. We denote by $[M, \mu]$ the image of $(M, \mu)$ in $K^{1}(A)$.

If $1_{M}$ is the identity automorphism of $M$, then trivially

$$
\left[M, 1_{M}\right]=0,\left[M, \mu^{-1}\right]=-[M, \mu] .
$$

Thus every element of $K^{1}(A)$ is of the form $[M, \mu]$ for some $M$ and some automorphism $\mu$ thereof.

If $A$ is a direct sum of the rings $A_{1}, \cdots, A_{n}$, then clearly

$$
K^{1}(A) \cong K^{1}\left(A_{1}\right) \oplus \cdots \oplus K^{1}\left(A_{n}\right) .
$$

5. Let $F$ be a field, and let $F^{*}$ be the multiplicative group of nonzero elements of $F$. For an $F$-module $V$, an automorphism $\phi$ of $V$ is just a nonsingular linear transformation on $V$. Let det $\phi$ denote the determinant of this transformation. We have $K^{1}(F) \cong F^{*}$, where $K^{1}(F)$ is written additively, $F^{*}$ multiplicatively. The isomorphism is given by $[V, \phi] \rightarrow \operatorname{det} \phi$.

Now suppose that $A$ is a full matrix algebra over $F$, and let $X$ be a fixed irreducible $A$-module. Each $A$-module is isomorphic to $X^{(n)}$ for some $n$, where $X^{(n)}$ denotes the direct sum of $n$ copies of $X$. Furthermore, $\operatorname{Hom}_{A}(X, X) \cong F$. Hence if $M=X^{(n)}$, and if $\mu$ is an automorphism of $M$, then $\mu$ may be represented by a nonsingular $n \times n$ matrix $T(\mu)$ with entries in $F$. The categories of $A$-modules and $F$-modules are isomorphic, and we have also

$$
K^{1}(A) \cong F^{*}
$$

the isomorphism being given by $[M, \mu] \rightarrow \operatorname{det} T(\mu)$.

2. Algebras over noetherian domains. 1. Let $R$ be a noetherian commutative integral domain, with quotient field $F$. If $M$ is a torsion free $R$-module, we may form the $F$-module $F \otimes_{R} M$, denoted by $F M$ for brevity. Let $A$ be an $R$-algebra which is finitely generated and torsion free as $R$-module, and set $A^{*}=F A$, an $F$-algebra. 
The additive groups $K^{0}(A), K^{0}\left(A^{*}\right)$ and $K^{1}\left(A^{*}\right)$ have already been defined in $\$ 1$. If $\left\{X_{1}^{*}, \cdots, X_{n}^{*}\right\}$ is a full set of irreducible $A^{*}$-modules, then $K^{0}\left(A^{*}\right)$ is just the free $Z$-module with Z-basis $\left[X_{1}^{*}\right], \cdots,\left[X_{n}^{*}\right]$.

2. Let $C_{f}$ denote the category of $R$-torsion-free $A$-modules. If we restrict ourselves to this category, we obtain a Grothendieck group $K_{f}^{0}(A)$. To each $M \in C_{f}$ corresponds an element $[M]_{f} \in K_{f}^{0}(A)$. The proof of Lemma 1 , $\$ 1.3$, remains unchanged. Hence if $M, N \in C_{f}$, then $[M]_{f}=[N]_{f}$ in $K_{f}^{0}(A)$ if and only if there exist exact sequences (1) for some choice of $U, V, W \in C_{f}$.

Using a procedure due to Swan [4], we show at once that $K_{f}^{0}(A) \cong K^{0}(A)$. The desired isomorphism $K_{f}^{0}(A) \rightarrow K^{0}(A)$ is given by $[M]_{f} \rightarrow[M], M \in C_{f}$, and the inverse map $\eta_{0}: K^{0}(A) \rightarrow K_{f}^{0}(A)$ may be obtained as follows: Let $M$ be any $A$-module, and choose an exact sequence

$$
0 \rightarrow X \rightarrow Y \rightarrow M \rightarrow 0
$$

with $Y$ a projective $A$-module. Then $X$ and $Y$ are in $C_{f}$, and we define

$$
\eta_{0}[M]=[Y]_{f}-[X]_{f} \text {. }
$$

By Schanuel's lemma, the image $\eta_{0}[M]$ is independent of the choice of $X$ and $Y$.

It is easily seen that if

$$
0 \rightarrow U \rightarrow V \rightarrow M \rightarrow 0
$$

is exact, with $U, V \in C_{f}$, then also

$$
\eta_{0}[M]=[V]_{f}-[U]_{f} .
$$

3. To each $M \in C_{f}$ there corresponds an $A^{*}$-module $F M$. It is easily verified that the map $[M]_{f} \rightarrow[F M]$ gives a mapping $\theta$ of $K_{f}^{0}(A)$ onto $K^{0}\left(A^{*}\right)$.

4. Next, we introduce the category $C_{t}$ of all $R$-torsion $A$-modules. If we restrict ourselves to this category, we obtain a Grothendieck group $K_{t}^{0}(A)$. To each $M \in C_{t}$ corresponds an element $[M]_{t} \in K_{t}^{0}(A)$. Since each short exact sequence from $C_{t}$ is a short exact sequence of $A$-modules, the map $[M]_{t} \rightarrow[M]$ gives a mapping of $K_{t}^{0}(A)$ into $K^{0}(A)$. Composing this map with the map $\eta_{0}$ defined above, we obtain a mapping $\eta: K_{t}^{0}(A) \rightarrow K_{f}^{0}(A)$. Indeed, if $M \in C_{t}$, choose any exact sequence (4) with $U, V \in C_{f}$, and then

$$
\eta\left([M]_{t}\right)=[V]_{f}-[U]_{f} .
$$

5. Suppose hereafter that $A^{*}$ is semisimple. Following Swan [4], we show the exactness of

$$
K_{t}^{0}(A) \stackrel{\eta}{\longrightarrow} K_{f}^{0}(A) \stackrel{\theta}{\longrightarrow} K^{0}\left(A^{*}\right) \longrightarrow 0 .
$$

Indeed, it is trivial that $\theta \eta=0$. On the other hand, let $x \in \operatorname{ker} \theta$, and write $x=[M]_{f}-[N]_{f}$ for some $M, N \in C_{f}$. From $\theta x=0$ we obtain $[F M]=[F N]$ in $K^{0}\left(A^{*}\right)$. Since $A^{*}$ is semisimple, this implies that $F M \cong F N$. Replacing $N$ by a 
module isomorphic to it does not change $[N]_{f}$, so we may assume that $F M=F N$, and that $N \subset M$. But then $M / N$ is an $R$-torsion module, and there is an exact sequence

Therefore

$$
0 \rightarrow N \rightarrow M \rightarrow M / N \rightarrow 0 .
$$

$$
x=[M]_{f}-[N]_{f}=\eta\left([M / N]_{t}\right) \in \text { image of } \eta .
$$

This completes the proof of the exactness of (5).

6. Now let $M, N \in C_{f}$ be any modules for which $F M=F N$. Define

$$
[M / / N]=\left[\frac{M}{M \cap \cap N}\right]_{t}-\left[\frac{N}{M \cap N}\right]_{t} \in K_{t}^{0}(A),
$$

which is meaningful since $F(M \cap N)=F M=F N$. For any module $X \subset M \cap N$ such that $F X=F(M \cap N)$, we have

$$
\left[\frac{M}{M \cap N}\right]_{t}=\left[\frac{M}{X}\right]_{t}-\left[\frac{M \cap N}{X}\right]_{t},
$$

which readily implies that

$$
[M / / N]=\left[\frac{M}{X}\right]_{t}-\left[\frac{N}{X}\right]_{t} .
$$

Lemma 2. Let $L, M, N \in C_{f}$ be such that $F L=F M=F N$. Then $[L / / M]+[M / / N]=[L / / N]$.

Proof. Choose $X=L \cap M \cap N$. Then $[L / / M]=[L / X]_{t}-[M / X]_{t}$, with analogous formulas for $[M / / N]$ and $[L / / N]$. The result now follows from formula (7).

Lemma 3. Let there be given modules $L_{i}, M_{i}, N_{i} \in C_{f}$ and exact sequences

$$
0 \longrightarrow L_{i} \stackrel{\phi_{i}}{\longrightarrow} M_{i} \stackrel{\psi_{i}}{\longrightarrow} N_{i} \longrightarrow 0, \quad i=1,2 .
$$

Let $L_{i}^{*}=F L_{i}$, and so on. Suppose there exist isomorphisms $\lambda: L_{1}^{*} \cong L_{2}^{*}$, $\mu: M_{1}^{*} \cong M_{2}^{*}, v: N_{1}^{*} \cong N_{2}^{*}$ for which the following diagram is commutative:

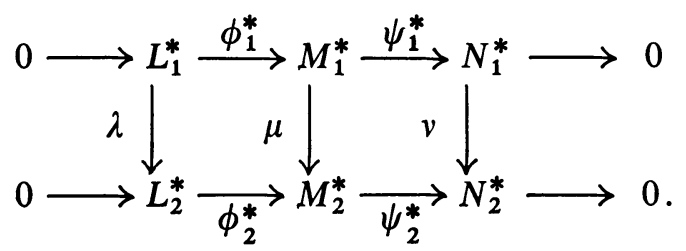

Then

$$
\left[M_{2} / / \mu M_{1}\right]=\left[L_{2} / / \lambda L_{1}\right]+\left[N_{2} / / v N_{1}\right] .
$$

Proof. The map $\phi_{2}^{*}$ induces a mapping $L_{2} \rightarrow M_{2} /\left(M_{2} \cap \mu M_{1}\right)$, and the kernel of this mapping is easily found to be $L_{2} \cap \lambda L_{1}$. Thus, there is an isomorphism of 
$L_{2} /\left(L_{2} \cap \lambda L_{1}\right)$ into $M_{2} /\left(M_{2} \cap \mu M_{1}\right)$. Analogously, there is a homomorphism of this latter module onto $N_{2} /\left(N_{2} \cap v N_{1}\right)$. A routine computation then shows the exactness of

$$
0 \rightarrow \frac{L_{2}}{L_{2} \cap \lambda L_{1}} \rightarrow \frac{M_{2}}{M_{2} \cap \mu M_{1}} \rightarrow \frac{N_{2}}{N_{2} \cap v N_{1}} \rightarrow 0 .
$$

Consequently

$$
\left[\frac{M_{2}}{M_{2} \cap \mu M_{1}}\right]_{t}=\left[\frac{L_{2}}{L_{2} \cap \lambda L_{1}}\right]_{t}+\left[\frac{N_{2}}{N_{2} \cap v N_{1}}\right]_{t} .
$$

An analogous formula holds with the numerators $M_{2}, L_{2}, N_{2}$ replaced by $\mu M_{1}$, $\lambda L_{1}, v N_{1}$, respectively. This implies the desired result.

7. We shall proceed to construct a homomorphism $\Delta: K^{1}\left(A^{*}\right) \rightarrow K_{t}^{0}(A)$. Using the notation of $\S 1.4$, write $K^{1}\left(A^{*}\right)=\mathscr{B} / \mathscr{B}_{0}$, and define

$$
\Delta\left(M^{*}, \mu^{*}\right)=\left[\mu^{*} M / / M\right],
$$

where $M \in C_{f}$ is chosen so that $F M=M^{*}$. Then $\Delta$ is well defined, since if also $F N=M^{*}, N \in C_{f}$, then

$$
\left[\mu^{*} M / / M\right]-\left[\mu^{*} N / / N\right]=\left[\mu^{*} M / / \mu^{*} N\right]-[M / / N]=0,
$$

the latter equality true because $\mu^{*}$ is an automorphism of $M^{*}$.

We now prove that $\Delta$ annihilates $\mathscr{B}_{0}$, and hence induces a map of $K^{1}\left(A^{*}\right)$ into $K_{t}^{0}(A)$. Consider first a generator of $\mathscr{B}_{0}$ of the form

$$
\left(M^{*}, \mu_{1}^{*} \mu_{2}^{*}\right)-\left(M^{*}, \mu_{1}^{*}\right)-\left(M^{*}, \mu_{2}^{*}\right) .
$$

Choose $M \in C_{f}$ such that $F M=M^{*}$. Then $\Delta$ maps the above generator onto

$$
\left[\mu_{1}^{*} \mu_{2}^{*} M / / M\right]-\left[\mu_{1}^{*} M / / M\right]-\left[\mu_{2}^{*} M / / M\right],
$$

which is zero because $\left[\mu_{1}^{*} \mu_{2}^{*} M / / \mu_{1}^{*} M\right]=\left[\mu_{2}^{*} M / / M\right]$.

Second, consider a generator of $\mathscr{B}_{0}$ of the form

where

$$
b_{0}=\left(M^{*}, \mu^{*}\right)-\left(L^{*}, \lambda^{*}\right)-\left(N^{*}, v^{*}\right),
$$

$$
0 \longrightarrow\left(L^{*}, \lambda^{*}\right) \stackrel{\phi}{\longrightarrow}\left(M^{*}, \mu^{*}\right) \stackrel{\psi}{\longrightarrow}\left(N^{*}, v^{*}\right) \longrightarrow 0
$$

is exact. Let $M \in C_{f}$ be such that $F M=M^{*}$, and set $L=\phi^{-1} M, N=\psi M$. Then we have the exact sequence

$$
0 \longrightarrow L \stackrel{\phi}{\longrightarrow} M \stackrel{\psi}{\longrightarrow} N \longrightarrow 0,
$$

which (when tensored with $F$ ) gives the exact sequence

$$
0 \longrightarrow L^{*} \stackrel{\phi}{\longrightarrow} M^{*} \stackrel{\psi}{\longrightarrow} N^{*} \longrightarrow 0 .
$$


Since the sequence (8) is exact, there is a commutative diagram

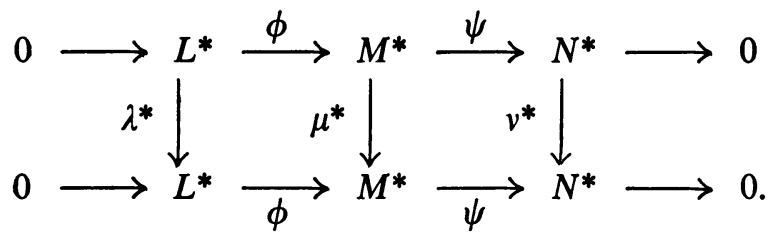

By Lemma 3, §2.7, we have

$$
\left[\mu^{*} M / / M\right]-\left[\lambda^{*} L / / L\right]-\left[v^{*} N / / N\right]=0 .
$$

But the left-hand side of the above equation is precisely $\Delta\left(b_{0}\right)$, which completes the proof that $\Delta\left(\mathscr{B}_{0}\right)=0$.

We shall use the same symbol $\Delta$ to denote the map of $K^{1}\left(A^{*}\right)$ into $K_{t}^{0}(A)$.

8. Let us now prove the exactness of

$$
K^{1}\left(A^{*}\right) \stackrel{\Delta}{\longrightarrow} K_{t}^{0}(A) \stackrel{\eta}{\longrightarrow} K_{f}^{0}(A) \stackrel{\theta}{\longrightarrow} K^{0}\left(A^{*}\right) \longrightarrow 0 .
$$

To begin with, we verify that $\eta \Delta=0$. For let $\left[M^{*}, \mu^{*}\right] \in K^{1}(A)$, and choose $M \in C_{f}$ such that $F M=M^{*}$. By definition,

$$
\Delta\left[M^{*}, \mu^{*}\right]=\left[\mu^{*} M / / M\right] .
$$

Choose $X=M \cap \mu^{*} M$, so that $F X=F M$. Then

$$
\begin{aligned}
\eta\left[\mu^{*} M / / M\right] & =\eta\left\{\left[\frac{\mu^{*} M}{X}\right]_{t}-\left[\frac{M}{X}\right]_{t}\right\} \\
& =\left[\mu^{*} M\right]_{f}-[X]_{f}-[M]_{f}+[X]_{f} \in K_{f}^{0}(A) \\
& =0
\end{aligned}
$$

since $\mu^{*} M \cong M$.

On the other hand, let us show that $\operatorname{ker} \eta \subset$ image of $\Delta$. For let $x \in \operatorname{ker} \eta$, and write $x=[M]_{t}-[N]_{t}$ for $M, N \in C_{t}$. Choose exact sequences

$$
0 \rightarrow X^{\prime} \rightarrow X \rightarrow M \rightarrow 0, \quad 0 \rightarrow Y^{\prime} \rightarrow Y \rightarrow N \rightarrow 0,
$$

with $X, X^{\prime}, Y, Y^{\prime} \in C_{f}$. Then

$$
0=\eta x=[X]_{f}-\left[X^{\prime}\right]_{f}-[Y]_{f}+\left[Y^{\prime}\right]_{f},
$$

so $\left[X \oplus Y^{\prime}\right]_{f}=\left[X^{\prime} \oplus Y\right]_{f}$ in $K_{f}^{0}(A)$. By $\S 2.2$, there exist modules $U, V, W \in C_{f}$ and exact sequences

$$
0 \rightarrow U \rightarrow X \oplus Y^{\prime} \oplus W \rightarrow V \rightarrow 0, \quad 0 \rightarrow U \rightarrow X^{\prime} \oplus Y \oplus W \rightarrow V \rightarrow 0 .
$$


Tensoring with $F$, and setting $U^{*}=F U, V^{*}=F V$, we obtain the exact sequences

$$
\begin{aligned}
& 0 \rightarrow U^{*} \rightarrow F\left(X \oplus Y^{\prime} \oplus W\right) \rightarrow V^{*} \rightarrow 0, \\
& 0 \rightarrow U^{*} \rightarrow F\left(X^{\prime} \oplus Y \oplus W\right) \rightarrow V^{*} \rightarrow 0 .
\end{aligned}
$$

But all short exact sequences of $A^{*}$-modules must split, since $A^{*}$ is assumed semisimple, and so there is an automorphism $\mu^{*}$ of $F\left(X \oplus Y^{\prime} \oplus W\right)$ for which the following diagram is commutative.

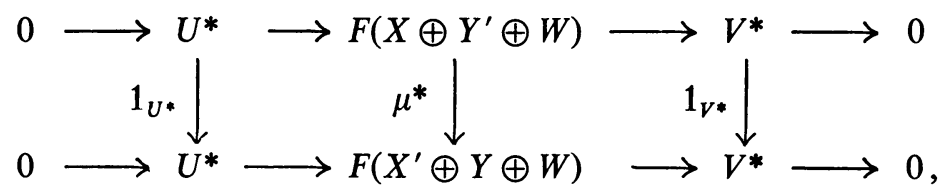

the 1's denoting identity maps. Since the restriction of an identity map is again an identity map, it now follows from Lemma $3, \S 2.6$, that

$$
\left[\mu^{*}\left(X \oplus Y^{\prime} \oplus W\right) / /\left(X^{\prime} \oplus Y \oplus W\right)\right]=0 .
$$

But then

$$
\left[\left(X \oplus Y^{\prime} \oplus W\right) / / \mu^{*}\left(X \oplus Y^{\prime} \oplus W\right)\right]=\left[\left(X \oplus Y^{\prime} \oplus W\right) / /\left(X^{\prime} \oplus Y \oplus W\right)\right] .
$$

Now the left-hand expression lies in the image of $\Delta$, while that on the right is equal to

$$
\left[\frac{X \oplus Y^{\prime} \oplus W}{X^{\prime} \oplus Y^{\prime} \oplus W}\right]_{t}-\left[\frac{X^{\prime} \oplus Y \oplus W}{X^{\prime} \oplus Y^{\prime} \oplus W}\right]_{t}=\left[\frac{X}{X^{\prime}}\right]_{t}-\left[\frac{Y}{Y^{\prime}}\right]_{t}=[M]_{t}-[N]_{t}=x
$$

This completes the proof that the sequence (9) is exact.

3. Group rings. 1. In this section we choose $R$ as a Dedekind domain of characteristic 0 , with quotient field $F$. (For example, $R$ might be the ring of all algebraic integers in an algebraic number field $F$.) Let $G$ be a finite group, and set $A=R G$, its group ring. Assume throughout this section that $F$ is a splitting field for $G$, so that $A^{*}(=F G)$ is a direct sum of full matrix algebras over $F$. We may choose $A$-modules $Z_{1}, \cdots, Z_{n} \in C_{f}$ (the category of $R$-torsion-free $A$-modules) such that if we set $Z_{i}^{*}=F Z_{i}$, then $\left\{Z_{1}^{*}, \cdots, Z_{n}^{*}\right\}$ is a full set of irreducible $A^{*}$. modules.

2. Let $P$ be a (nonzero) prime ideal of $R$, and set $K=R / P, A=A / P A$. Then $\bar{A}$ is a $\bar{K}$-algebra, and $K^{0}(\bar{A})$ is a free $Z$-module with free $Z$-basis $\left[\bar{Y}_{1}\right], \cdots,\left[\bar{Y}_{m}\right]$, where $\bar{Y}_{1}, \cdots, \bar{Y}_{m}$ are a full set of irreducible $A$-modules.

The decomposition numbers $d_{i j}^{P}$ are non-negative integers such that $\bar{Y}_{j}$ occurs with multiplicity $d_{i j}^{P}$ as composition factor of the $A$-module $Z_{i} / P Z_{i}$. Therefore

$$
\left[\frac{Z_{i}}{P Z_{i}}\right]=\sum_{j} d_{i j}^{P}\left[Y_{j}\right] \text { in } K^{0}(A), \quad 1 \leqq i \leqq n
$$


When $P$ does not divide the order of $G$, the decomposition matrix $\left(d_{i j}^{P}\right.$, is just the identity matrix.

For arbitrary $P$, Brauer $[2 ; 3]$ has shown that $m \leqq n$, and that the G.C.D. of the $m \times m$ minors of the decomposition matrix $\left(d_{i j}^{P}\right)$ is equal to 1 . Therefore we may solve equations $(10)$ for the $\left[\bar{Y}_{j}\right]$ in terms of the $\left[Z_{i} / P Z_{i}\right]$, and so there exist rational integers $e_{i j}^{P}$ (not necessarily unique) such that

$$
\left[\bar{Y}_{j}\right]=\sum_{i} e_{i j}^{P}\left[\frac{Z_{i}}{P Z_{i}}\right] \text { in } K^{0}(\tilde{A}), \quad 1 \leqq j \leqq m .
$$

Furthermore, we have $\left[Z_{i} / / P^{k} Z_{i}\right]=k\left[Z_{i} / P Z_{i}\right]$ in $K^{0}(\bar{A})$, for each rational integer $k$. Therefore every element of $K^{0}(\bar{A})$ is expressible as a sum

$$
\sum_{i=1}^{n}\left[P^{k_{i}} Z_{i} / / Z_{i}\right]
$$

3. Now let $P$ range over the prime ideals of $R$, and as in $\S 2$, let $K_{t}^{0}(A)$ be the Grothendieck group of the category of $R$-torsion $A$-modules. Since each such module is a direct sum of its $P$-primary components, we have

$$
K_{t}^{0}(A) \cong \sum_{P}^{\oplus} K^{0}\left(\frac{A}{P A}\right)
$$

Hence, using the results of the preceding paragraph, every element of $K_{t}^{0}(A)$ is expressible as a sum

$$
\sum_{i=1}^{n}\left[J_{i} Z_{i} / / Z_{i}\right], \quad J_{i}=\text { fractional } R \text {-ideal in } K .
$$

4. We set $\mathscr{J}=$ multiplicative group of fractional $R$-ideals in $K$, and let $\mathscr{J}^{n}=\mathscr{J} \times \cdots \times \mathscr{J}$ ( $n$ factors). Then there is a homomorphism $\tau: \mathscr{J}^{n} \rightarrow K_{t}^{0}(A)$ given by

$$
\tau\left(J_{1}, \cdots, J_{n}\right)=\left[J_{1} Z_{1} / / Z_{1}\right]+\cdots+\left[J_{n} Z_{n} / / Z_{n}\right],
$$

and we have just shown that $\tau$ is a surjection.

Using the notation of the exact sequence (9), let us set $\sigma=\eta \tau$. Then

$$
\sigma\left(J_{1}, \cdots, J_{n}\right)=\sum_{i=1}^{n}\left\{\left[J_{i} Z_{i}\right]_{f}-\left[Z_{i}\right]_{f}\right\}
$$

and the kernel of $\theta$ equals the image of $\eta$, which in turn equals the image of $\sigma$. Now $K^{0}\left(A^{*}\right)$ is a free $Z$-module, so by the exactness of $(9)$, we have

$$
K_{f}^{0}(A) \cong K^{0}\left(A^{*}\right) \oplus \text { image of } \sigma,
$$

the above being an isomorphism of additive groups. Furthermore, image of $\sigma \cong \mathscr{J}^{n} / \operatorname{ker} \sigma$. 
Thus, to compute the additive structure of $K_{f}^{0}(A)$, it suffices to determine $\operatorname{ker} \sigma$. We shall compute this kernel explicitly.

5. If $R$ is a principal ideal ring, then each $J_{i} \in \mathscr{J}$ is of the form $R a_{i}$ for some $a_{i} \in F$, and thus

$$
\left[J_{i} Z_{i}\right]_{f}=\left[a_{i} Z_{i}\right]_{f}=\left[Z_{i}\right]_{f},
$$

since $a_{i} Z_{i} \cong Z_{i}$. In this case we see that the image of $\sigma$ is 0 , and so $K_{f}^{0}(A) \cong K^{0}\left(A^{*}\right)$ as additive groups.

6. If $R$ is not necessarily a principal ideal ring, the above argument still shows that the kernel of $\sigma$ contains $\mathscr{J}_{0}^{n}$, defined as

$$
\mathscr{J}_{0}^{n}=\left\{\left(J_{1}, \cdots, J_{n}\right) \in \mathscr{J}^{n}: \text { each } J_{i} \text { is principal }\right\} .
$$

We now make use of the decomposition matrices $\left(d_{i j}^{P}\right)$ defined in $\$ 3.2$. When $P$ divides the order of $G$, the matrix $\left(d_{i j}^{p}\right)$ is not a square matrix, and so there exist rational integers $q_{1}, \cdots, q_{n}$ (not all zero) such that $\sum_{i} q_{i} d_{i j}^{P}=0$ for all $j$. But then

Set

$$
\sum_{i}\left[Z_{i} / / P^{q_{i}} Z_{i}\right]=\sum_{i} q_{i}\left[\frac{Z_{i}}{P Z_{i}}\right]=\sum_{i, j} q_{i} d_{i j}^{P}\left[\bar{Y}_{j}\right]=0 \text { in } K_{t}^{0}(A) \text {. }
$$

$$
D_{P}=\left\{\left(P^{q_{1}}, \cdots, P^{q_{n}}\right) \in \mathscr{J}^{n}: \sum_{t} q_{i} d_{i j}^{P}=0 \text { for all } j\right\} .
$$

Then the preceding remarks imply that $\tau\left(D_{P}\right)=0$ for each $P$. Indeed, since $K_{t}^{0}(A) \cong \Sigma^{\oplus} K^{0}(A / P A)$, we have shown that

$$
\operatorname{ker} \tau=\prod_{P} D_{P}
$$

Note that $D_{P}=\{1\}$ whenever $P$ does not divide the order of $G$.

5. Next, from the relation $\sigma=\eta \tau$ we conclude that $\operatorname{ker} \sigma \supset \operatorname{ker} \tau$. Combining this fact with the observation of $\S 3.6$, we have

$$
\operatorname{ker} \sigma \supset \mathscr{J}_{0}^{n} \cdot \operatorname{ker} \tau .
$$

We shall now prove that in fact

$$
\operatorname{ker} \sigma=\mathscr{J}_{0}^{n} \cdot \operatorname{ker} \tau .
$$

To begin with, since $F$ is a splitting field for $G$, we may write $A^{*}=A_{1}^{*} \oplus \cdots \oplus A_{n}^{*}$ where each $A_{i}^{*}$ is a full matrix algebra over $F$. For each $i$, the $A^{*}$-module $Z_{i}^{*}$ is then an irreducible $A_{i}^{*}$-module. Let $F^{*}$ be the multiplicative group of the field $F$. By the discussion of $\S 1.5$, we have

$$
K^{1}\left(A^{*}\right) \cong \sum_{i=1}^{n}{ }^{\otimes} K^{1}\left(A_{i}^{*}\right) \cong F^{*} \times \cdots \times F^{*}(n \text { factors }) .
$$

We may thus define a map $\rho: K^{1}\left(A^{*}\right) \rightarrow \mathscr{J}^{n}$ by

$$
\rho\left(a_{1}, \cdots, a_{n}\right)=\left(R a_{1}, \cdots, R a_{n}\right), \quad a_{i} \in F^{*} .
$$


Indeed, $a_{1}$ (as element of $K^{1}\left(A_{1}^{*}\right)$ ) represents the pair $\left[Z_{1}^{*}, a_{1}^{*}\right]$, where $a_{1}^{*}$ is the automorphism $z \rightarrow a_{1} z, z \in Z_{1}^{*}$. Then

$$
\begin{aligned}
\Delta\left[Z_{1}^{*}, a_{1}^{*}\right] & =\left[a_{1} Z_{1} / / Z_{1}\right]=\tau\left(R a_{1}, R, \cdots, R\right) \\
& =\tau \rho\left(a_{1}, 1, \cdots, 1\right) .
\end{aligned}
$$

Corresponding results hold for $a_{2}, \cdots, a_{n}$, which shows that $\Delta=\tau \rho$. We therefore have a commutative diagram

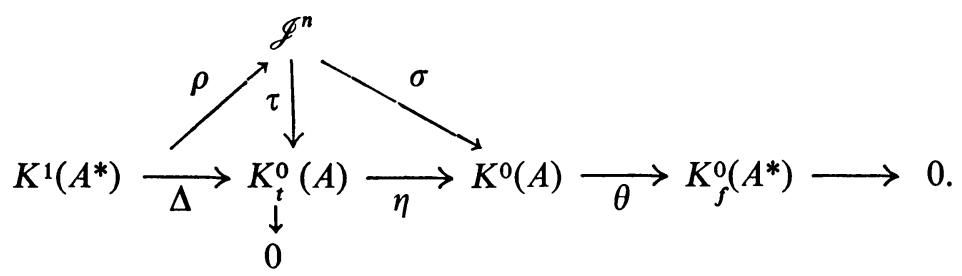

Using this diagram, a routine argument shows that $\operatorname{ker} \sigma=(\operatorname{ker} \tau)$ (image of $\rho$ ). However, the image of $\rho$ is precisely the group $\mathscr{J}_{0}^{n}$ defined in \$3.6. This completes the proof of formula (12), and so we have determined the structure of $K_{f}^{0}(A)$ (and thus of $K_{0}(A)$ ) as additive group.

6. Let us investigate briefly what happens in the nonsplitting field case. Let $R_{0}$ be the ring of all algebraic integers in an algebraic number field $F_{0}$, and set $A_{0}=R_{0} G, A_{0}^{*}=F_{0} G$. The semisimple algebra $A_{0}^{*}$ need not be a direct sum of full matrix algebras. Nevertheless, there is an exact sequence

$$
K^{1}\left(A_{0}^{*}\right) \rightarrow K_{t}^{0}\left(A_{0}\right) \rightarrow K_{f}^{0}\left(A_{0}\right) \stackrel{\theta_{0}}{\longrightarrow} K^{0}\left(A_{0}^{*}\right) \rightarrow 0,
$$

so again

$$
K_{f}^{0}\left(A_{0}\right) \cong K^{0}\left(A_{0}^{*}\right) \oplus \operatorname{ker} \theta_{0}
$$

as additive groups. We shall show that $\operatorname{ker} \theta_{0}$ is a finite abelian group.

To begin with, we observe that $K_{f}^{0}\left(A_{0}\right)$ is finitely generated as $Z$-module. For let $V_{1}^{*}, \cdots, V_{s}^{*}$ be a full set of irreducible $A_{0}^{*}$-modules. For each $i$, consider the set of $A_{0}$-modules $W$ which are $R_{0}$-torsion-free and satisfy $F_{0} W=V_{i}^{*}$. By the Jordan-Zassenhaus theorem, there are only a finite number of nonisomorphic $A_{0}$-modules in this set, say $W_{i 1}, \cdots, W_{i t_{i}}$. But then it is easily seen that the elements

$$
\left\{\left[W_{i j}\right]_{f} \in K_{f}^{0}\left(A_{0}\right): 1 \leqq j \leqq t_{i}, \quad 1 \leqq i \leqq s\right\}
$$

are a set of generators of the $Z$-module $K_{f}^{0}\left(A_{0}\right)$. (They are surely not a $Z$-basis, however.)

It follows then that $\operatorname{ker} \theta_{0}$ is also finitely generated as $Z$-module, so we need only show that $\operatorname{ker} \theta_{0}$ is a torsion module. We begin by choosing a finite extension $F$ of $F_{0}$ which is a splitting field for $G$, say $\left(F: F_{0}\right)=k$. Let $R$ be the integral closure of $R_{0}$ in $F$; then $R$ is a Dedekind ring with quotient field $F$, and we have 


$$
R \cong R_{0} \oplus \cdots \oplus R_{0} \oplus J \quad \text { ( } k \text { summands) }
$$

as $R_{0}$-modules, where $J$ is some ideal in $R_{0}$.

For each $R_{0}$-torsion-free $A_{0}$-module $M$, define $\alpha[M]=\left[R \otimes_{R_{0}} M\right]$, thereby obtaining a map $\alpha: K_{f}^{0}\left(A_{0}\right) \rightarrow K_{f}^{0}(A)$. Analogously, there is a map $\alpha^{*}: K^{0}\left(A_{0}^{*}\right)$ $\rightarrow K^{0}\left(A^{*}\right)$. On the other hand, every $A$-module can be viewed as an $A_{0}$-module, so there are maps $\beta: K_{f}^{0}(A) \rightarrow K_{f}^{0}\left(A_{0}\right), \beta^{*}: K^{0}\left(A^{*}\right) \rightarrow K^{0}\left(A_{0}^{*}\right)$, and we have a commutative diagram

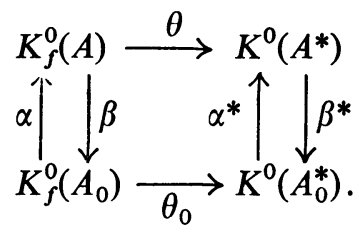

Let $x \in \operatorname{ker} \theta_{0}$; then $\alpha x \in \operatorname{ker} \theta$, so there exists a positive integer $q$ such that $q \cdot \alpha x=0$, and therefore $q \cdot \beta \alpha x=0$. However,

$$
\beta \alpha[M]=\beta\left[R \otimes_{R_{0}} M\right]=(k-1)[M]+[J M] \text { in } K_{f}^{0}\left(A_{0}\right) .
$$

Choose a positive integer $h$ such that $J^{h}$ is principal. Then the above implies that $h \cdot \beta \alpha[M]=h k[M]$, and thus

$$
0=h \cdot q \cdot \beta \alpha x=q h k x .
$$

This completes the proof that $\operatorname{ker} \theta_{0}$ is a finite abelian group. We shall not attempt to obtain an explicit computation for this group.

REMARK. Since $K^{1}$ is functorial the sequence (4) extends to a sequence

$$
K^{1}(A) \rightarrow K^{1}\left(A^{*}\right) \stackrel{\Delta}{\longrightarrow} K_{t}^{0}(A) \rightarrow K^{0}(A) \rightarrow K^{0}\left(A^{*}\right) \rightarrow 0 .
$$

This extended sequence is not in general exact. Indeed if $A=Z[t] /\left(t^{2}-1\right)$, the group ring of a group of order 2 , then $K^{1}\left(A^{*}\right) \cong Q^{*} \times Q^{*}$ and the kernel of $\Delta$ is $\left\{\left( \pm 2^{k}, \pm 2^{-k}\right)\right\}$. But $K^{1}(A)$ is easily seen to be just the four-group.

\section{REFERENCES}

1. H. Bass, K-theory and stable algebra, Inst. Hautes Etudes Publ. (to appear).

2. R. Brauer, On the Cartan invariants of groups of finite order, Ann. of Math. (2) 42 (1941), 53-61.

3. - A characterization of the characters of groups of finite order, Ann. of Math. (2) 57 (1953), 357-377.

4. R. G. Swan, Induced representations and projective modules, Ann, of Math. (2) 71 (1960), 552-578.

5. - The Grothendieck ring of a finite group, Topology 2 (1963), 85-110.

UNIVERSITY OF ILLINOIS,

Urbana, ILlinoIS 